\title{
Beam-size effect at colliders and correlations of particles in a beam
}

\author{
G. L. Kotkin and V. G. Serbo* \\ Novosibirsk State University, 630090, Novosibirsk, Russia
}

(Received 8 August 2003; published 4 October 2004)

\begin{abstract}
For several processes at colliding beams, macroscopically large impact parameters give an essential contribution to the standard cross section. These impact parameters may be much larger than the transverse sizes of the colliding bunches. In that case, the standard calculations have to be essentially modified. The corresponding formulas for such a beam-size effect were given 20 years ago without taking into account correlations of particle coordinates in the beams. In the present paper we derive formulas necessary to take into account quantitatively the effect of particle correlations in the spectrum of bremsstrahlung as well as in pair production. Our results are quite different from those obtained in recent papers [V. N. Baier and V. M. Katkov, Phys. Rev. D 66, 053009 (2002); V. N. Baier and V. M. Katkov, hep-ph/0305304.]. We point out the origin of this difference.
\end{abstract}

DOI: 10.1103/PhysRevSTAB.7.101001

PACS numbers: $13.66 .-\mathrm{a}$

\section{INTRODUCTION}

The so-called beam-size or MD effect is a phenomenon discovered in experiments at the MD-1 detector on the VEPP-4 collider, Novosibirsk 1981 (the term "MD effect" is used in honor of the MD-1 group). It was observed [1] that the number of measured photons in the process $e^{+} e^{-} \rightarrow e^{+} e^{-} \gamma$ was considerably smaller than expected. A qualitative explanation of the effect was given by Tikhonov [2], who pointed out that those impact parameters $\varrho$, which give an essential contribution to the standard cross section, reach values of $\varrho_{m} \sim 5 \mathrm{~cm}$ whereas the transverse size of the bunch is $\sigma_{\perp} \sim$ $10^{-3} \mathrm{~cm}$. The limitation of the impact parameters to values $\varrho \lessgtr \sigma_{\perp}$ is just the reason for the decreasing number of observed photons. The first calculations of this effect have been performed in Refs. [3,4]. The detailed description of the MD effect can be found in Ref. [5]. Later on, the effect of limited impact parameters was taken into account using the single bremsstrahlung reaction for measuring the luminosity at the VEPP-4 collider [6] and at the LEP-I collider [7].

A general scheme to calculate the finite beam-size effect was developed in Ref. [8] starting from the quantum description of collisions as an interaction of wave packets that form bunches. Since the effect under discussion is dominated by small momentum transfer, the general formulas can be considerably simplified. The corresponding approximate formulas were derived in [8]. In the second step, the transverse motion of the particles in the beams can be neglected. The less exact (but simpler) formulas, which are then found, correspond to the results of Refs. [3,4]. It has also been shown that similar effects have to be expected for several other reactions such as bremsstrahlung for colliding $e p$ beams $[9,10], e^{+} e^{-}$pair production in $e^{ \pm} e$, and $\gamma e$ collisions [8].

*Electronic address: serbo@math.nsc.ru
The corresponding corrections to the standard formulas are now included in programs for simulation of events at linear colliders. The influence of MD effect on polarization was considered in Ref. [11]. In 1995 the MD effect was experimentally observed at the electron-proton collider HERA [12] at the level predicted in [10].

The possibility of creating high-energy colliding $\mu^{+} \mu^{-}$beams is now widely discussed. For several processes at such colliders a new type of beam-size effect will take place - the so-called linear beam-size effect [13]. The calculation of this effect was performed by methods developed for the MD effect in [8].

It was realized in recent years that the MD effect in bremsstrahlung plays an important role in the beam lifetime problem. At storage rings TRISTAN and LEP-I, the process of single bremsstrahlung was the dominant mechanism for the particle losses in beams. If an electron loses more than $1 \%$ of its energy, it leaves the beam. Since the MD effect considerably reduced the effective cross section of this process, the calculated beam lifetime in these storage rings was larger by about $25 \%$ for TRISTAN [14] and by about $40 \%$ for LEP-I [15] (in accordance with the experimental data) than without taking into account the MD effect. According to our calculations [16], at B factories PEP-II and KEK-B the MD effect reduces beam losses due to bremsstrahlung by about $20 \%$.

It is seen from this brief listing that the MD effect is a phenomenon interesting from the theoretical point of view and important from the experimental point of view. In the present paper we consider once again the MD effect to take into account correlations of particle coordinates in the beams. Usually these correlations are small; however, more accurate measurements may be sensitive to them. In the present paper we derive formulas which are necessary to take into account quantitatively the effect of particle correlations in the spectrum of bremsstrahlung as well as in pair production. 
Additionally, we compare our results with those obtained in recent papers $[17,18]$ in which previous results $[3,4,8]$ about bremsstrahlung spectrum were revised. It was claimed that an additional subtraction, related to the coherent contribution, has to be done. In the present paper we analyze the coherent and incoherent contributions in the conditions, considered in papers $[17,18]$, when the coherent length is much smaller than the bunch length but much larger than the mean distance between particles. In the present paper we derive expressions for the coherent and incoherent contributions and show that under these conditions the coherent contribution is completely negligible and, therefore, there is no need to revise the previous results. Our preliminary results were given in [19].

In the next section we present the qualitative description of the MD effect. In Sec. III we discuss our approximations. Basic formulas for coherent and incoherent contributions are given in Sec. IV. Corrections to the standard cross section, related to the particle correlations, are derived in Sec. V. A comparison of our results with those in Refs. [17,18] is presented in Sec. VI. Some conclusions are given in Sec. VII.

\section{QUALITATIVE DESCRIPTION OF THE MD EFFECT}

Qualitatively we describe the MD effect using the $e p \rightarrow e p \gamma$ process as an example. [Below we use the following notations: $N_{e}$ and $N_{p}$ are the numbers of electrons and protons (positrons) in the bunches, $\sigma_{z}=l$ is the longitudinal, $\sigma_{x}$ and $\sigma_{y}$ are the horizontal and vertical transverse sizes of the proton (positron) bunch, $\gamma_{e}=$ $E_{e} /\left(m_{e} c^{2}\right), \gamma_{p}=E_{p} /\left(m_{p} c^{2}\right)$, and $r_{e}=e^{2} /\left(m_{e} c^{2}\right)$ is the classical electron radius.] This reaction is described by the diagram in Fig. 1 which corresponds to the radiation of the photon by the electron (the contribution of the photon radiation by the proton can be neglected). The large impact parameters $\varrho \gtrsim \sigma_{\perp}$, where $\sigma_{\perp}$ is the transverse beam size, correspond to small momentum transfer $\hbar q_{\perp} \sim(\hbar / \varrho) \lesssim\left(\hbar / \sigma_{\perp}\right)$. In this region, the given reaction can be represented as a Compton scattering (Fig. 2) of an equivalent photon, radiated by the proton, on the electron. The equivalent photons with frequency $\omega$ form a "disk" of radius $\varrho_{m} \sim \gamma_{p} c / \omega$, where $\gamma_{p}=E_{p} /\left(m_{p} c^{2}\right)$ is the Lorentz factor of the proton. Indeed, the electromagnetic

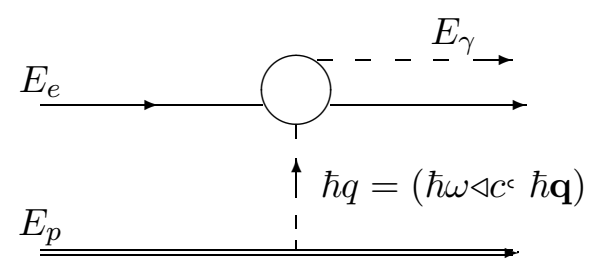

FIG. 1. Block diagram of radiation by the electron.

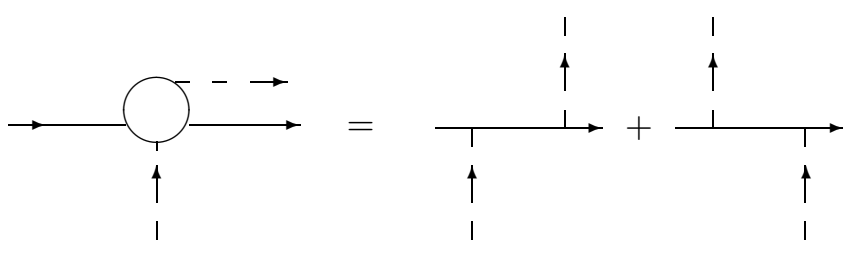

FIG. 2. Compton scattering of equivalent photon on the electron.

field of the proton is $\gamma_{p}$ times contracted in the direction of motion. Therefore, at distance $\varrho$ from the axis of motion a characteristic longitudinal length of a region occupied by the field can be estimated as $\lambda \sim \varrho / \gamma_{p}$, which leads to the frequency $\omega \sim c / \lambda \sim \gamma_{p} c / \varrho$.

In the frame of reference connected with the collider, the equivalent photon with energy $\hbar \omega$ and the electron with energy $E_{e} \gg \hbar \omega$ move toward each other (Fig. 3) and perform the Compton scattering. The characteristics of this process are well known. The main contribution to the Compton scattering is given by the region where the scattered photons fly in a direction opposite that of the initial photons. For such a backward scattering, the energy of the equivalent photon $\hbar \omega$, the energy of the final photon $E_{\gamma}$, and its emission angle $\theta_{\gamma}$ are related by

$$
\hbar \omega=\frac{E_{\gamma}}{4 \gamma_{e}^{2}\left(1-E_{\gamma} / E_{e}\right)}\left[1+\left(\gamma_{e} \theta_{\gamma}\right)^{2}\right]
$$

and, therefore, for typical emission angles $\theta_{\gamma} \lesssim 1 / \gamma_{e}$ one has

$$
\hbar \omega \sim \frac{E_{\gamma}}{4 \gamma_{e}^{2}\left(1-E_{\gamma} / E_{e}\right)} .
$$

As a result, we find the radius of the disk of equivalent photons with the frequency $\omega$ (corresponding to a final photon with energy $E_{\gamma}$ ) as follows:

$$
\begin{aligned}
& \varrho_{m}=\frac{\gamma_{p} c}{\omega} \sim 4 \lambda_{e} \gamma_{e} \gamma_{p} \frac{E_{e}-E_{\gamma}}{E_{\gamma}}, \\
& \lambda_{e}=\frac{\hbar}{m_{e} c}=3.86 \times 10^{-11} \mathrm{~cm} .
\end{aligned}
$$

For the HERA collider with $E_{p}=820 \mathrm{GeV}$ and $E_{e}=$ $28 \mathrm{GeV}$ one obtains

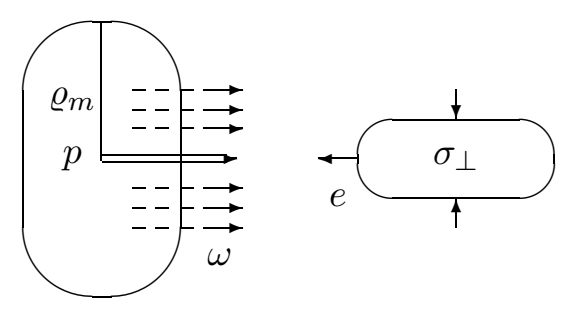

FIG. 3. Scattering of equivalent photons, forming the disk with radius $\varrho_{m}=\gamma_{p} c / \omega$, on the electron beam with radius $\sigma_{\perp}$. 


$$
\varrho_{m} \gtrsim 1 \mathrm{~cm} \text { for } E_{\gamma} \lesssim 0.2 \mathrm{GeV} .
$$

Equation (3) is also valid for the $e^{-} e^{+} \rightarrow e^{-} e^{+} \gamma$ process with replacement of the protons by the positrons. For the VEPP-4 collider it leads to

$$
\varrho_{m} \gtrsim 1 \mathrm{~cm} \text { for } E_{\gamma} \lesssim 15 \mathrm{MeV},
$$

for the KEK-B and PEP-II colliders we have

$$
\varrho_{m} \gtrsim 1 \mathrm{~cm} \text { for } E_{\gamma} \lesssim 0.1 \mathrm{GeV} .
$$

The standard calculation corresponds to the interaction of the photons (that form the disk) with the unbounded flux of electrons. However, the particle beams at the HERA collider have finite transverse beam sizes of the order of $\sigma_{\perp} \sim 10^{-2} \mathrm{~cm}$. Therefore, the equivalent photons from the region $\sigma_{\perp} \lesssim \varrho \lessgtr \varrho_{m}$ cannot interact with the electrons from the other beam. This leads to the reduction of the number of the observed photons. The "observed cross section" $d \sigma_{\mathrm{obs}}$ is smaller than the standard cross section $d \sigma$ calculated for an infinite transverse extension of the electron beam,

$$
d \sigma_{\mathrm{obs}}=d \sigma-d \sigma_{\mathrm{cor}} .
$$

Here the correction $d \sigma_{\text {cor }}$ can be presented in the form

$$
d \sigma_{\mathrm{cor}}=d \sigma_{\mathrm{C}}\left(\omega, E_{e}, E_{\gamma}\right) d n(\omega),
$$

where $d n(\omega)$ denotes the number of "missing" equivalent photons and $d \sigma_{\mathrm{C}}$ is the cross section of the Compton scattering. Let us stress that the equivalent photon approximation in this region has a high accuracy (the neglected terms are of the order of $1 / \gamma_{p}$ ). But for the qualitative description it is sufficient to use the logarithmic approximation in which this number is (see [20], Sec. 99)

$$
d n=\frac{\alpha}{\pi} \frac{d \omega}{\omega} \frac{d q_{\perp}^{2}}{q_{\perp}^{2}}
$$

Since $q_{\perp} \sim 1 / \varrho$, we can present the number of missing equivalent photons in another form,

$$
d n=\frac{\alpha}{\pi} \frac{d \omega}{\omega} \frac{d \varrho^{2}}{\varrho^{2}},
$$

with the integration region in $\varrho$ :

$$
\sigma_{\perp} \lesssim \varrho \lesssim \varrho_{m}=\frac{\gamma_{p} c}{\omega} .
$$

As a result, this number is equal to

$$
d n(\omega)=2 \frac{\alpha}{\pi} \frac{d \omega}{\omega} \ln \frac{\varrho_{m}}{\sigma_{\perp}},
$$

and the correction to the standard cross section with logarithmic accuracy is

$$
\begin{aligned}
d \sigma_{\mathrm{cor}} & =\frac{16}{3} \alpha r_{e}^{2} \frac{d y}{y}\left(1-y+\frac{3}{4} y^{2}\right) \ln \frac{4 \gamma_{e} \gamma_{p}(1-y) \lambda_{e}}{y \sigma_{\perp}}, \\
y & =\frac{E_{\gamma}}{E_{e}} .
\end{aligned}
$$

[Within this approximation, the standard cross section has the form

$$
\begin{aligned}
d \sigma & =d \sigma_{\mathrm{C}} \frac{\alpha}{\pi} \frac{d \omega}{\omega} \frac{d q_{\perp}^{2}}{q_{\perp}^{2}} \\
& =\frac{16}{3} \alpha r_{e}^{2} \frac{d y}{y}\left(1-y+\frac{3}{4} y^{2}\right) \ln \frac{4 \gamma_{e} \gamma_{p}(1-y)}{y}
\end{aligned}
$$

with the integration region $\omega /\left(c \gamma_{p}\right) \lesssim q_{\perp} \lesssim m_{e} c / \hbar$ corresponding to the impact parameters $\varrho$ in the interval $\lambda_{e} \lesssim \varrho \lesssim \varrho_{m}$.]

\section{APPROXIMATIONS}

For future linear $e^{+} e^{-}$colliders the transverse sizes of the beams will change significantly during the time of interaction due to a mutual attraction of very dense beams. However, for most of the ordinary accelerators, including practically all $e^{+} e^{-}$and $e p$ storage rings, the change of the transverse beam sizes during the collisions can be neglected. Below we use two main approximations: (i) the particle movement in the bunches has a quasiclassical character, and (ii) the particle distribution remains practically unchanged during the collision. For definiteness, we use again the $e p$ collision as an example.

Therefore, if the proton (electron) bunch moves along (opposite) the direction of $z$ axis with the velocity $v_{p}$ $\left(v_{e}\right)$, its density has the form

$$
n_{p}=n_{p}\left(\boldsymbol{\varrho}, z-v_{p} t\right), \quad n_{e}=n_{e}\left(\boldsymbol{\varrho}, z+v_{e} t\right) .
$$

We also introduce so-called "transverse densities"

$$
n_{p}(\boldsymbol{\varrho})=\int n_{p} d z, \quad n_{e}(\boldsymbol{\varrho})=\int n_{e} d z
$$

which is equal to the total number of protons (electrons) which cross a unit area around the impact parameter $\varrho$ during the collision. Using the transverse densities, we express the luminosity for collisions of beams whose axes are separated by the impact parameter $\varrho$ as

$$
L(\varrho)=\int n_{e}\left(\mathbf{r}_{\perp}\right) n_{p}\left(\varrho+\mathbf{r}_{\perp}\right) d^{2} \mathbf{r}_{\perp} .
$$

The usual luminosity for a single collision of $e p$ beams $L_{e p}$ is then

$$
L_{e p}=L(0) \text {. }
$$

Below we consider in detail the case when an electron deflection angle $\theta_{e}$ is smaller than the typical radiation angle $\sim 1 / \gamma_{e}$. It is easy to estimate the ratio of these angles. The electric $\mathbf{E}$ and magnetic $\mathbf{B}$ fields of the proton bunch are approximately equal in magnitude, $|\mathbf{E}| \approx$ 
$|\mathbf{B}| \sim e N_{p} /\left[\left(\sigma_{x}+\sigma_{y}\right) l\right]$. These fields are transverse and they deflect the electron into the same direction. In such fields the electron moves around a circumference of radius $R \sim \gamma_{e} m_{e} c^{2} /(e B)$ and gets the deflection angle $\theta_{e} \sim$ $l / R$. Therefore, the ratio of these angles is of the order of

$$
\frac{\theta_{e}}{\left(1 / \gamma_{e}\right)} \sim \eta=\frac{r_{e} N_{p}}{\sigma_{x}+\sigma_{y}} \text {. }
$$

The parameter $\eta \gg 1$ only for the SLC and future linear $e^{+} e^{-}$colliders (see the TESLA, NLC, JLC, and CLIC projects), in most of the existing storage rings $\eta \lesssim 1$.

In our consideration we use the equivalent photon approximation. In the region of interest (where impact parameters are large, $\varrho \geq \sigma_{\perp}$ ) this simple and transparent method has a high accuracy: the neglected terms are of the order of $1 / \gamma$. It should be stressed that the operator quasiclassical method, used in Ref. [17], just coincides in this region with the equivalent photon approximation.

\section{COHERENT AND INCOHERENT CONTRIBUTIONS}

\section{A. General formulas}

The corresponding formulas for the number of events in a single collision of the electron and proton bunches can be found in Refs. [21,22]. To calculate the MD effect, we need to know the distribution of equivalent photons (EP) for large values of impact parameters. In this region we can consider the electron-proton scattering as the scattering of electrons on the electromagnetic field of the proton bunch. Replacing this field by the flux of EP with some frequency distribution, we obtain the number of events in the form

$$
\begin{aligned}
& d N=d L_{\gamma e}(\omega) d \sigma_{\mathrm{C}}\left(\omega, E_{e}, E_{\gamma}\right), \\
& d L_{\gamma e}(\omega)=n_{\gamma}(\varrho, \omega) d \omega n_{e}(\varrho) d^{2} \varrho
\end{aligned}
$$

Here $n_{e}(\boldsymbol{\varrho})$ is the transverse electron density (15) and $n_{\gamma}(\boldsymbol{\varrho}, \omega) d \omega$ is the transverse density of EP with the frequencies in the interval from $\omega$ to $\omega+d \omega$. The quantity $d L_{\gamma e}(\omega)$ denotes the differential luminosity for the collisions of EP and electrons and $d \sigma_{\mathrm{C}}\left(\omega, E_{e}, E_{\gamma}\right)$ is the Compton cross section for the scattering of the equivalent photon with the frequency $\omega$ on the electron.

For comparison with the experimental data the number of events in a single collision of beams $d N$ should be averaged over many collisions of bunches in a given experiment. For example, the typical rate at the HERA collider is less than $1 / 100$ bremsstrahlung photons in a certain interval of frequencies per a single collision of the beams; therefore, in that experiment the averaging over many collisions of bunches really does exist.

The transverse density of the EP is determined by density of the electromagnetic field for a given frequency, i.e., by $\left|\mathbf{E}_{\omega}(\boldsymbol{\varrho})\right|^{2} /(4 \pi)$, where $\mathbf{E}_{\omega}(\boldsymbol{\varrho})$ is the spectral component of the collective electric field of the proton bunch.
As a result, the transverse density of the EP is

$$
n_{\gamma}(\boldsymbol{\varrho}, \omega) d \omega=\frac{c}{4 \pi^{2}}\left\langle\left|\mathbf{E}_{\omega}(\boldsymbol{\varrho})\right|^{2}\right\rangle \frac{d \omega}{\hbar \omega},
$$

where the sign $\langle\cdots\rangle$ denotes the above mentioned statistical averaging. The field $\mathbf{E}_{\omega}(\boldsymbol{\varrho})$ itself depends on a distribution of charges in the proton bunch at $t=0$. We introduce the exact (fluctuating) density of the proton bunch $n(\mathbf{r})$ and the average density

$$
n_{p}(\mathbf{r})=\langle n(\mathbf{r})\rangle
$$

as well as the corresponding form factor

$$
F_{p}(\mathbf{q})=\int n_{p}(\mathbf{r}) e^{-i \mathbf{q r}} d^{3} r
$$

with the normalization

$$
F_{p}(0)=\int n_{p}(\mathbf{r}) d^{3} r=N_{p}
$$

In the classical limit

$$
n(\mathbf{r})=\sum_{a} \delta\left(\mathbf{r}-\mathbf{r}_{a}\right),
$$

where $\mathbf{r}_{a}$ is the radius vector of the $a$ th proton. In these notations, the exact (fluctuating) collective field is

$$
\begin{aligned}
\mathbf{E}_{\omega}(\varrho) & =-\frac{i e}{\pi c} \int d^{2} q_{\perp} \frac{\mathbf{q}_{\perp} e^{i \mathbf{q}_{\perp} \varrho}}{D(\mathbf{q})} \int d^{3} r n(\mathbf{r}) e^{-i \mathbf{q} \mathbf{r}}, \\
D(\mathbf{q}) & =\mathbf{q}_{\perp}^{2}+\frac{q_{z}^{2}}{\gamma_{p}^{2}}
\end{aligned}
$$

with $q_{z}=\omega / c$.

As a result, the number of events

$$
\begin{aligned}
d N & \propto n_{\gamma}(\boldsymbol{\varrho}, \omega) \\
& =\frac{\alpha}{4 \pi^{4} \omega} \int \frac{\left(\mathbf{q}_{\perp} \mathbf{q}_{\perp}^{\prime}\right)}{D(\mathbf{q}) D\left(\mathbf{q}^{\prime}\right)} e^{i\left(\mathbf{q}_{\perp}-\mathbf{q}_{\perp}^{\prime}\right)} \boldsymbol{\varrho} S\left(\mathbf{q}, \mathbf{q}^{\prime}\right) d^{2} q_{\perp} d^{2} q_{\perp}^{\prime}
\end{aligned}
$$

depends on the beam structure factor

$$
\begin{aligned}
S\left(\mathbf{q}, \mathbf{q}^{\prime}\right) & =\int S\left(\mathbf{r}, \mathbf{r}^{\prime}\right) e^{-i\left(\mathbf{q r}-\mathbf{q}^{\prime} \mathbf{r}^{\prime}\right)} d^{3} r d^{3} r^{\prime}, \\
S\left(\mathbf{r}, \mathbf{r}^{\prime}\right) & =\left\langle n(\mathbf{r}) n\left(\mathbf{r}^{\prime}\right)\right\rangle
\end{aligned}
$$

in which

$$
q_{z}=q_{z}^{\prime}=\omega / c .
$$

Below we analyze these formulas in conditions when the coherence length $l_{\text {coh }} \sim c / \omega$ is much smaller than the bunch length $l$, but much larger that the mean distance between particles in the beam $a$, i.e., at

$$
a \ll \frac{c}{\omega} \sim l_{\mathrm{coh}}=\frac{4 \gamma_{e}^{2} \hbar c}{E_{\gamma}}\left(1-E_{\gamma} / E_{e}\right) \ll l .
$$




\section{B. The beam structure factor}

The obtained general formulas include the coherent and incoherent contributions. The coherent contribution is determined by the average field which is given by Eq. (25) with the replacement of the exact density $n(\mathbf{r})$ by the average density $n_{p}(\mathbf{r})$. The average density of the proton bunch has a single scale in the longitudinal direction - the length of the bunch $l$. Therefore, the average field of the bunch is essential in the region of frequencies $\omega=c q_{z} \lesssim c / l$ and should be small in the region of large frequencies $\omega \gg c / l$. In particular, if the proton bunch has the Gaussian distribution,

$$
n_{p}(\mathbf{r})=\frac{N_{p}}{(2 \pi)^{3 / 2} \sigma_{x} \sigma_{y} l} \exp \left[-\frac{x^{2}}{2 \sigma_{x}^{2}}-\frac{y^{2}}{2 \sigma_{y}^{2}}-\frac{z^{2}}{2 l^{2}}\right]
$$

its form factor is equal to

$$
F_{p}(\mathbf{q})=N_{p} \exp \left[-\frac{1}{2}\left(q_{x} \sigma_{x}\right)^{2}-\frac{1}{2}\left(q_{y} \sigma_{y}\right)^{2}-\frac{1}{2}(\omega l / c)^{2}\right]
$$

and vanishes in the discussed region of frequencies from the interval (29).

If we introduce the density fluctuation

$$
\Delta n(\mathbf{r})=n(\mathbf{r})-n_{p}(\mathbf{r}),
$$

we can rewrite the average product of densities in the form

$$
\left\langle n(\mathbf{r}) n\left(\mathbf{r}^{\prime}\right)\right\rangle=n_{p}(\mathbf{r}) n_{p}\left(\mathbf{r}^{\prime}\right)+\left\langle\Delta n(\mathbf{r}) \Delta n\left(\mathbf{r}^{\prime}\right)\right\rangle .
$$

In accordance with this presentation, we split the function $S\left(\mathbf{r}, \mathbf{r}^{\prime}\right)$ in two items called coherent and incoherent contribution:

$$
\begin{aligned}
& S=S_{\text {coh }}+S_{\text {incoh }}, \quad S_{\text {coh }}\left(\mathbf{r}, \mathbf{r}^{\prime}\right)=n_{p}(\mathbf{r}) n_{p}\left(\mathbf{r}^{\prime}\right), \\
& S_{\text {incoh }}\left(\mathbf{r}, \mathbf{r}^{\prime}\right)=\left\langle\Delta n(\mathbf{r}) \Delta n\left(\mathbf{r}^{\prime}\right)\right\rangle .
\end{aligned}
$$

The coherent contribution to the structure factor is equal to

$$
S_{\mathrm{coh}}\left(\mathbf{q}, \mathbf{q}^{\prime}\right)=F_{p}(\mathbf{q}) F_{p}^{*}\left(\mathbf{q}^{\prime}\right) .
$$

This formula was used in Refs. [21,22] to obtain main characteristics of the coherent bremsstrahlung. It also allows us to obtain the following estimate for the Gaussian beam in the region of interest (at $\left|q_{x}\right|,\left|q_{x}^{\prime}\right| \lesssim$ $1 / \sigma_{x}$ and $\left.\left|q_{y}\right|,\left|q_{y}^{\prime}\right| \lesssim 1 / \sigma_{y}\right)$ :

$$
S_{\text {coh }}\left(\mathbf{q}, \mathbf{q}^{\prime}\right) \sim N_{p}^{2} \exp \left[-(\omega l / c)^{2}\right] .
$$

Let us now consider the incoherent contribution. A bunch at colliders can be treated as a continuous media with a smooth average particle distribution. It was shown in the appendix that for such a media the function $S_{\text {incoh }}\left(\mathbf{r}, \mathbf{r}^{\prime}\right)$ is expressed only via the average density $n_{p}(\mathbf{r})$ and via the correlation function $C\left(\mathbf{r}, \mathbf{r}^{\prime}\right)$ as follows:

$$
S_{\text {incoh }}\left(\mathbf{r}, \mathbf{r}^{\prime}\right)=\delta\left(\mathbf{r}-\mathbf{r}^{\prime}\right) n_{p}(\mathbf{r})+C\left(\mathbf{r}, \mathbf{r}^{\prime}\right)
$$

If we neglect the correlations of the particle coordinates in the beam, the correlation function $C\left(\mathbf{r}, \mathbf{r}^{\prime}\right)$ vanishes, and we obtain [taking into account Eq. (28)]

$$
S_{\text {incoh }}\left(\mathbf{q}, \mathbf{q}^{\prime}\right)=F\left(\mathbf{q}_{\perp}-\mathbf{q}_{\perp}^{\prime}\right) .
$$

It is important that this expression is determined only by the transverse average density of the proton bunch and it does not depend on $\omega$. Equation (38) has been used to derive the previous results about MD effect (for details see Ref. [5] and Sec. V). For the Gaussian beam in the region of interest, we get from (38) a useful estimate

$$
S_{\text {incoh }}\left(\mathbf{q}, \mathbf{q}^{\prime}\right) \sim N_{p} .
$$

The correlations of the particle coordinates may arise due to Coulomb interaction of particles in the beam. In this case the characteristic quantity-the correlation length $l_{\text {correl }}$-is related to the Debye radius. It is evident that the correlations are negligible if the correlation length is much larger than the coherence length, i.e., at $l_{\text {correl }} \gg l_{\text {coh }}$. According to an estimate [4] it is just the case for the VEPP-4 experiment [1]. In any case, the correlations may give an essential correction to the standard bremsstrahlung cross section only if

$$
l_{\text {correl }} \lesssim l_{\text {coh. }} \text {. }
$$

Therefore, the important quantity is the spectral component of the correlation function:

$$
C_{\omega}\left(\mathbf{r}_{\perp}, \mathbf{r}_{\perp}^{\prime}\right)=\int C\left(\mathbf{r}, \mathbf{r}^{\prime}\right) e^{-i \omega\left(z-z^{\prime}\right) / c} d z d z^{\prime}
$$

With this notation the incoherent contribution is now

$$
S_{\text {incoh }}\left(\mathbf{q}, \mathbf{q}^{\prime}\right)=F\left(\mathbf{q}_{\perp}-\mathbf{q}_{\perp}^{\prime}\right)+C_{\omega}\left(\mathbf{q}_{\perp}, \mathbf{q}_{\perp}^{\prime}\right),
$$

where

$$
C_{\omega}\left(\mathbf{q}_{\perp}, \mathbf{q}_{\perp}^{\prime}\right)=\int C_{\omega}\left(\mathbf{r}_{\perp}, \mathbf{r}_{\perp}^{\prime}\right) e^{-i\left(\mathbf{q}_{\perp} \mathbf{r}_{\perp}-\mathbf{q}_{\perp}^{\prime} \mathbf{r}_{\perp}^{\prime}\right)} d^{2} r_{\perp} d^{2} r_{\perp}^{\prime} .
$$

\section{CORRECTION TO THE STANDARD BREMSSTRAHLUNG CROSS SECTION}

Let us compare the coherent and incoherent contributions for the Gaussian beams. In this case, the ratio

$$
\frac{d N^{\mathrm{coh}}}{d N^{\text {incoh }}} \sim \frac{S_{\mathrm{coh}}\left(\mathbf{q}, \mathbf{q}^{\prime}\right)}{S_{\text {incoh }}\left(\mathbf{q}, \mathbf{q}^{\prime}\right)} \sim N_{p} \exp \left[-(\omega l / c)^{2}\right]
$$

is determined by the parameter $\omega l / c$. Since $\hbar \omega \sim$ $E_{\gamma} /\left[4 \gamma_{e}^{2}\left(1-E_{\gamma} / E_{e}\right)\right]$, it is also useful to introduce the coherence length (29) and the critical energy for coherent bremsstrahlung 


$$
E_{c}=\frac{4 \gamma_{e}^{2} \hbar c}{l}
$$

If the coherence length is large, $l_{\text {coh }} \gtrsim l$, or if the final photon energy is small, $E_{\gamma} \lesssim E_{c}$, the parameter $\omega l / c \lesssim 1$ and the coherent contribution is dominant.

On the contrary, in the region of large photon energy, $E_{\gamma} \gg E_{c}$, or small coherence length, $l_{\text {coh }} \ll l$, considered here, the incoherent contribution dominates. In particular, for $N_{p} \sim 10^{11}$ the ratio $d N^{\mathrm{coh}} / d N^{\text {incoh }}$ is small even for $\omega l / c=6$,

$$
\frac{d N^{\mathrm{coh}}}{d N^{\mathrm{incoh}}} \sim N_{p} e^{-36} \ll 1
$$

and the coherent contribution becomes completely negligible. In this case the number of events for bremsstrahlung can be presented in the form [cf. (7)]

$$
d N^{\mathrm{incoh}}=L_{e p} d \sigma_{\mathrm{obs}}, \quad d \sigma_{\mathrm{obs}}=d \sigma-d \sigma_{\mathrm{cor}},
$$

where $L_{e p}$ is the luminosity (17) of the $e p$ collisions, $d \sigma$ is the standard cross section for the $e p \rightarrow e p \gamma$ process, and $d \sigma_{\text {cor }}$ is the correction related to the MD effect. Then we perform integration over $\mathbf{q}_{\perp}$ and $\mathbf{q}_{\perp}^{\prime}$ using the wellknown equality

$$
\int \frac{\mathbf{q}_{\perp} e^{i \mathbf{q}_{\perp} \varrho}}{\mathbf{q}_{\perp}^{2}+(1 / b)^{2}} d^{2} q_{\perp}=\frac{2 \pi i}{b} \frac{\varrho}{\varrho} K_{1}(\varrho / b)
$$

where $K_{n}(x)$ denotes the modified Bessel function of the third kind (the McDonald function). As a result, we obtain the correction to the standard cross section in the form [cf. with the approximate formulas (8) and (12)]

$$
d \sigma_{\mathrm{cor}}=d \sigma_{\mathrm{C}}\left(\omega, E_{e}, E_{\gamma}\right) \frac{\alpha}{\pi} \frac{d \omega}{\omega} G(\omega)
$$

where $d \sigma_{\mathrm{C}}$ is the Compton cross section and the function $G(\omega)$ consists of two items

$$
G(\omega)=G^{(1)}(\omega)+G^{(2)}(\omega)
$$

The first item represents the previous result for the MD effect (without taking into account the correlations),

$$
\begin{aligned}
& G^{(1)}(\omega)=\int \frac{d^{2} \varrho}{\pi \varrho_{m}^{2}}\left[1-\frac{L(\varrho)}{L(0)}\right] K_{1}^{2}\left(\varrho / \varrho_{m}\right), \\
& \varrho_{m}=\frac{c \gamma_{p}}{\omega}
\end{aligned}
$$

where $L(\varrho)$ is defined in (16). Some other useful expressions for $G^{(1)}(\omega)$ as well as its asymptotics can be found in [5]. The second item is directly related to the correlation function (37) and (41)

$$
\begin{aligned}
G^{(2)}(\omega)= & -\int \frac{d^{2} \varrho}{\pi \varrho_{m}^{2}} \frac{n_{e}(\varrho)}{L_{e p}} C_{\omega}\left(\mathbf{r}_{\perp}+\varrho, \mathbf{r}_{\perp}^{\prime}+\varrho\right) \\
& \times \frac{\left(\mathbf{r}_{\perp} \mathbf{r}_{\perp}^{\prime}\right)}{r_{\perp} r_{\perp}^{\prime}} K_{1}\left(r_{\perp} / \varrho_{m}\right) K_{1}\left(r_{\perp}^{\prime} / \varrho_{m}\right) d^{2} r_{\perp} d^{2} r_{\perp}^{\prime} .
\end{aligned}
$$

Note that the main contribution to $G^{(1)}(\omega)(51)$ is given by the region of large impact parameters (11) while the main contribution to $G^{(2)}(\omega)$ is given by the region $\varrho \lesssim \sigma_{\perp}$. If the quantity $\varrho_{m}$ is small, $\varrho_{m} \ll \sigma_{\perp}$, the correction to the standard cross section vanishes, since in this case $K_{1}\left(\varrho / \varrho_{m}\right) \sim \varrho_{m} / \varrho$ and $G(\omega) \sim\left(\varrho_{m} / \sigma_{\perp}\right)^{2} \ll 1$.

The quantity $d \sigma_{\mathrm{C}} d \omega / \omega$ in (49) can be expressed via the energy $E_{\gamma}$ and the emission angle $\theta_{\gamma}$ of the final photon as follows [taking into account relation (1)]:

$$
\begin{aligned}
d \sigma_{\mathrm{C}} \frac{\alpha}{\pi} \frac{d \omega}{\omega} & =2 \alpha r_{e}^{2} \frac{d y}{y} \frac{d z}{(1+z)^{2}} F(y, z), \\
F(y, z) & =2(1-y) \frac{1+z^{2}}{(1+z)^{2}}+y^{2},
\end{aligned}
$$

where

$$
y=\frac{E_{\gamma}}{E_{e}}, \quad z=\left(\theta_{\gamma} \gamma_{e}\right)^{2}, \quad r_{e}=\frac{e^{2}}{m_{e} c^{2}} .
$$

\section{COMPARISON WITH THE RESULTS OF REFS. [17,18]}

We derive the final expression for the incoherent contribution from general equations (19), (20), and (25) as a simple consequence of natural assumptions about the particle distribution in a proton bunch. It is useful to rewrite these equations in the form convenient for comparison with the corresponding equations in $[17,18]$. To do this, we note that the Compton cross section $d \sigma_{\mathrm{C}} \propto$ $\left|\mathbf{e} \mathbf{M}_{\text {Compton }}\right|^{2}$, where $\mathbf{e} \mathbf{M}_{\text {Compton }}$ is the amplitude of the Compton scattering for the EP with the polarization vector $\mathbf{e}=\mathbf{E}_{\omega}(\boldsymbol{\varrho}) /\left|\mathbf{E}_{\omega}(\boldsymbol{\varrho})\right|$. Therefore, the number of events in a given collision of beams is proportional to $|M|^{2}$, where

$$
M=\mathbf{E}_{\omega}(\boldsymbol{\varrho}) \mathbf{M}_{\text {Compton }}
$$

is related to the probability amplitude of the process. Further, we use Eqs. (24) and (48) and present the collective field of the proton bunch $\mathbf{E}_{\omega}(\boldsymbol{\varrho})$ as a sum of fields of all protons:

$$
\begin{aligned}
\mathbf{E}_{\omega}(\boldsymbol{\varrho}) & =\sum_{a=1}^{N_{p}} \mathbf{E}_{\omega}^{(a)}(\boldsymbol{\varrho}), \\
\mathbf{E}_{\omega}^{(a)}(\boldsymbol{\varrho}) & =\frac{2 e}{c \varrho_{m}} \frac{\boldsymbol{\varrho}_{a}^{\prime}}{\varrho_{a}^{\prime}} K_{1}\left(\varrho_{a}^{\prime} / \varrho_{m}\right) e^{-i \omega z_{a} / c},
\end{aligned}
$$

where $\varrho_{a}^{\prime}=\boldsymbol{\varrho}-\boldsymbol{\varrho}_{a}$ is the impact parameter between the 
electron and the ath proton, and the parameter $\varrho_{m}=$ $\gamma_{p} c / \omega$ is the radius of the disk of EP (see Fig. 3).

As a consequence, the amplitude $M$ is the sum

$$
\begin{aligned}
M & =\sum_{a=1}^{N_{p}} m_{a} e^{-i \omega z_{a} / c}, \\
m_{a} & =\frac{2 e}{c \varrho_{m}} K_{1}\left(\varrho_{a}^{\prime} / \varrho_{m}\right) \frac{\varrho_{a}^{\prime}}{\varrho_{a}^{\prime}} \mathbf{M}_{\mathrm{Compton}},
\end{aligned}
$$

where the item $m_{a} \exp \left(-i \omega z_{a} / c\right)$ is the contribution to $M$ related to the interaction of the electron with the ath proton, while $|M|^{2}$ can be presented as a double sum

$$
|M|^{2}=\sum_{a, b} m_{a} m_{b}^{*} e^{-i \omega\left(z_{a}-z_{b}\right) / c} .
$$

We split this sum into the sum with $a=b$ and the sum with $a \neq b$ :

$$
\begin{aligned}
|M|^{2} & =\Sigma_{1}+\Sigma_{2}, \quad \Sigma_{1}=\sum_{a}\left|m_{a}\right|^{2}, \\
\Sigma_{2} & =\sum_{a \neq b} m_{a} m_{b}^{*} e^{-i \omega\left(z_{a}-z_{b}\right) / c} .
\end{aligned}
$$

Equations (57)-(59) can be considered as the same starting formulas in our approach, based on the equivalent photon approximation, and in the approach of $[17,18]$, based on the operator quasiclassical method.

However, further calculations are quite different. As in Refs. [17,18], below we consider the case when one can separate the transverse and longitudinal distributions and neglect the correlations between the transverse coordinates in the proton bunch. It means that we can present the exact $n(\mathbf{r})$ and average $n_{p}(\mathbf{r})$ densities in the form

$$
n(\mathbf{r})=f\left(\mathbf{r}_{\perp}\right) n(z), \quad\langle n(\mathbf{r})\rangle \equiv n_{p}(\mathbf{r})=f_{p}\left(\mathbf{r}_{\perp}\right) n_{p}(z)
$$

with the normalizations

$$
\int f\left(\mathbf{r}_{\perp}\right) d^{2} r_{\perp}=1, \quad \int n(z) d z=N_{p} .
$$

In the classical limit one has

$$
\begin{aligned}
n(z) & =\sum_{a} \delta\left(z-z_{a}\right), \\
F_{p}\left(q_{z}\right) & =\int n_{p}(z) e^{-i q_{z} z} d z=\sum_{a}\left\langle e^{-i q_{z} z_{a}}\right\rangle,
\end{aligned}
$$

where $z_{a}$ is the longitudinal coordinate of the $a$ th proton. It was shown in the appendix that the average product of the longitudinal densities $\left\langle n(z) n\left(z^{\prime}\right)\right\rangle$ is expressed only via the average longitudinal density and the longitudinal correlation function $C\left(z, z^{\prime}\right)$ as follows [cf. (33)-(37)]:

$$
\begin{aligned}
& \left\langle n(z) n\left(z^{\prime}\right)\right\rangle=n_{p}(z) n_{p}\left(z^{\prime}\right)+\left\langle\Delta n(z) \Delta n\left(z^{\prime}\right)\right\rangle, \\
& \left\langle\Delta n(z) \Delta n\left(z^{\prime}\right)\right\rangle=\delta\left(z-z^{\prime}\right) n_{p}(z)+C\left(z, z^{\prime}\right) .
\end{aligned}
$$

In our approach, the number of events is proportional to $|M|^{2}$ averaged over collisions of beams, i.e.,

$$
d N \propto\left\langle|M|^{2}\right\rangle=\left\langle\Sigma_{1}\right\rangle+\left\langle\Sigma_{2}\right\rangle .
$$

Here the item $\left\langle\Sigma_{1}\right\rangle$ contains the factor $\left\langle\left|m_{a}\right|^{2}\right\rangle_{\perp}$ and the item $\left\langle\Sigma_{2}\right\rangle$ contains the factor $\left\langle m_{a}\right\rangle_{\perp}\left\langle m_{b}^{*}\right\rangle_{\perp}$ where

$$
\begin{aligned}
\left\langle\left|m_{a}\right|^{2}\right\rangle_{\perp} & =\int\left|m_{a}\right|^{2} f_{p}\left(\boldsymbol{\varrho}_{a}\right) d^{2} \varrho_{a}, \\
\left\langle m_{a}\right\rangle_{\perp} & =\int m_{a} f_{p}\left(\boldsymbol{\varrho}_{a}\right) d^{2} \varrho_{a} .
\end{aligned}
$$

Both these factors do not depend on index $a$. Therefore, the first item is equal to

$$
\left\langle\Sigma_{1}\right\rangle=N_{p}\left\langle\left|m_{a}\right|^{2}\right\rangle_{\perp}
$$

that leads to the correction, corresponding to $G^{(1)}$ in (49) $-(51)$.

The second item is equal to

$$
\left\langle\Sigma_{2}\right\rangle=\left|\left\langle m_{a}\right\rangle_{\perp}\right|^{2}\langle Z\rangle, \quad Z=\sum_{a \neq b} e^{-i \omega\left(z_{a}-z_{b}\right) / c} .
$$

It was shown in the appendix that

$$
\langle Z\rangle=\left|F_{p}(\omega / c)\right|^{2}+C_{\omega},
$$

where the longitudinal form factor $F_{p}\left(q_{z}\right)$ is defined in (62) and the longitudinal structure factor is

$$
C_{\omega}=\int C\left(z, z^{\prime}\right) e^{-i \omega\left(z-z^{\prime}\right) / c} d z d z^{\prime}
$$

In the considered region of large frequencies (29), the longitudinal form factor is small, in particular, for the Gaussian beams [cf. (36), (44), and (46)]

$$
\left|F_{p}(\omega / c)\right|^{2}=N_{p}^{2} \exp \left[-(\omega l / c)^{2}\right] \ll N_{p} .
$$

As a result, we obtain

$$
\left\langle\Sigma_{2}\right\rangle=\left|\left\langle m_{a}\right\rangle_{\perp}\right|^{2} C_{\omega},
$$

that leads to the correction, corresponding to $G^{(2)}$ in (49)-(52) with

$$
C_{\omega}\left(\mathbf{r}_{\perp}, \mathbf{r}_{\perp}^{\prime}\right)=f_{p}\left(\mathbf{r}_{\perp}\right) f_{p}\left(\mathbf{r}_{\perp}^{\prime}\right) C_{\omega} .
$$

Therefore, in the considered approximation the correction $G^{(2)}$ has the form

$$
\begin{aligned}
G^{(2)}(\omega)= & -C_{\omega} \int \frac{d^{2} \varrho}{\pi \varrho_{m}^{2}} \frac{n_{e}(\varrho)}{L_{e p}}\left[\frac{\mathbf{r}_{\perp}}{r_{\perp}} K_{1}\left(r_{\perp} / \varrho_{m}\right)\right. \\
& \left.\times f_{p}\left(\mathbf{r}_{\perp}+\varrho\right) d^{2} r_{\perp}\right]^{2} .
\end{aligned}
$$

Usually the correlations of particles in beams are small. If we can neglect the correlations of the longitudinal coordinates in the proton bunch, the quantity $\langle Z\rangle$ becomes small, 


$$
|\langle Z\rangle| \ll N_{p}
$$

and the item $\left\langle\Sigma_{2}\right\rangle$, as well as $G^{(2)}(\omega)$, vanishes,

$$
\left|\left\langle\Sigma_{2}\right\rangle\right| \ll\left\langle\Sigma_{1}\right\rangle, \quad\left|G^{(2)}(\omega)\right| \ll G^{(1)}(\omega) .
$$

Let us consider the opposite case, when the fluctuations are absent,

$$
\left\langle\Delta n(z) \Delta n\left(z^{\prime}\right)\right\rangle=0
$$

In this unrealistic case a beam is similar to the crystal, in which particles are localized near the centers of the crystal structure. Now the correlations are large,

$$
C\left(z, z^{\prime}\right)=-\delta\left(z-z^{\prime}\right) n_{p}(z),
$$

the quantity $C_{\omega}$ becomes large as well

$$
C_{\omega}=-N_{p},
$$

and correction $G^{(2)}(\omega)$ reads

$$
\begin{aligned}
G^{(2)}(\omega)= & N_{p} \int \frac{d^{2} \varrho}{\pi \varrho_{m}^{2}} \frac{n_{e}(\varrho)}{L_{e p}}\left[\frac{\mathbf{r}_{\perp}}{r_{\perp}} K_{1}\left(r_{\perp} / \varrho_{m}\right)\right. \\
& \left.\times f_{p}\left(\mathbf{r}_{\perp}+\varrho\right) d^{2} r_{\perp}\right]^{2}
\end{aligned}
$$

The authors of $[17,18]$ as the first step had performed averaging over transverse coordinates of the protons. Certainly, after that they get the same expression for $\Sigma_{1}$ as in (67). For $\Sigma_{2}$ they had obtained the following expression:

$$
\left\langle\Sigma_{2}\right\rangle_{\perp}=\left|\left\langle m_{a}\right\rangle_{\perp}\right|^{2} Z
$$

When calculating $Z$, they add and subtract the items with $a=b$, as a consequence,

$$
Z=J-N_{p}, \quad J=\sum_{a, b} e^{-i \omega\left(z_{a}-z_{b}\right) / c}=\left|\sum_{a} e^{-i \omega z_{a} / c}\right|^{2} .
$$

Their next step consists of replacement of the sum $J$ by the integral

$$
J \rightarrow\left|\int e^{-i \omega z / c} n_{p}(\mathbf{r}) d^{3} r\right|^{2},
$$

which is negligible in the considered region. In particular, for the Gaussian beam the replacement given by Eq. (83) means the following [see (71)]:

$$
J \rightarrow N_{p}^{2} \exp \left[-(\omega l / c)^{2}\right] \ll N_{p} .
$$

This estimate leads to a large negative value of

$$
Z=-N_{p}
$$

and to

$$
\left\langle\Sigma_{2}\right\rangle_{\perp}=-N_{p}\left|\left\langle m_{a}\right\rangle_{\perp}\right|^{2} .
$$

As a result, the correction $G^{(2)}$ was obtained in the form of Eq. (80). Just the expression (80) is a new "subtraction term" derived in $[17,18]$.

It is clearly seen that results (76) and (80) are quite different. The origin of this difference is in the incorrect calculation of quantity $Z$ in Refs. [17,18], where this quantity was found to be a large negative value $Z=$ $-N_{p}$. It is not difficult to understand that in the considered region of large frequencies (29) the quantity $Z$ fluctuates near zero for various sets of coordinates $\left\{z_{a}\right\}=$ $z_{1}, z_{2}, \ldots, z_{N_{p}}$, corresponding to various collisions of beams. After averaging over many collisions, one obtains the estimate $|\langle Z\rangle| \ll N_{p}$. Moreover, let us stress that the dispersion of $Z$ is large, $\Delta Z \sim N_{p}$; therefore, the averaging over many various sets of $\left\{z_{a}\right\}$ is necessary to obtain the stable result for $\langle Z\rangle$. This natural behavior of $Z$ is illustrated by numerical calculations given below.

Let us consider in detail replacement (83) which leads to result $Z=-N_{p}$. This replacement is true for the region of small frequencies $\omega l / c \ll 1$ when $J=N_{p}^{2}$ and $Z=$ $-N_{p}+J=N_{p}\left(N_{p}-1\right) \approx N_{p}^{2}$, but such a replacement is completely incorrect in the considered region of large frequencies (29). To show this, we perform numerical calculation of the sum $J$. For a given collision of beams, we can consider a set of the longitudinal proton coordinates $\left\{z_{a}\right\}$ as a set of random quantities with some distribution $w(z)$. We assume below that

$$
w(z)=\frac{1}{\sqrt{2 \pi l}} \exp \left(-\frac{z^{2}}{2 l^{2}}\right)
$$

Now the sum

$$
\sum_{a=1}^{N_{p}} e^{-i q z_{a}}=C-i S
$$

with

$$
C=\sum_{a=1}^{N_{p}} \cos \left(q z_{a}\right), \quad S=\sum_{a=1}^{N_{p}} \sin \left(q z_{a}\right), \quad q=\omega / c
$$

is also the random quantity as well as

$$
J=C^{2}+S^{2} .
$$

The quantities $C$ and $S$ are the sums of large numbers of random items. Therefore, one can expect that they distribute in accordance with the normal law:

$$
\begin{aligned}
\frac{d W}{d C} & =\frac{1}{\sqrt{2 \pi N_{p}} \Delta c} \exp \left[-\frac{\left(C-N_{p} \bar{c}\right)^{2}}{2 N_{p}(\Delta c)^{2}}\right], \\
\frac{d W}{d S} & =\frac{1}{\sqrt{2 \pi N_{p} \bar{s}^{2}}} \exp \left[-\frac{S^{2}}{2 N_{p} \bar{s}^{2}}\right]
\end{aligned}
$$

where $\Delta c=\sqrt{\bar{c}^{2}-\bar{c}^{2}}$ and 


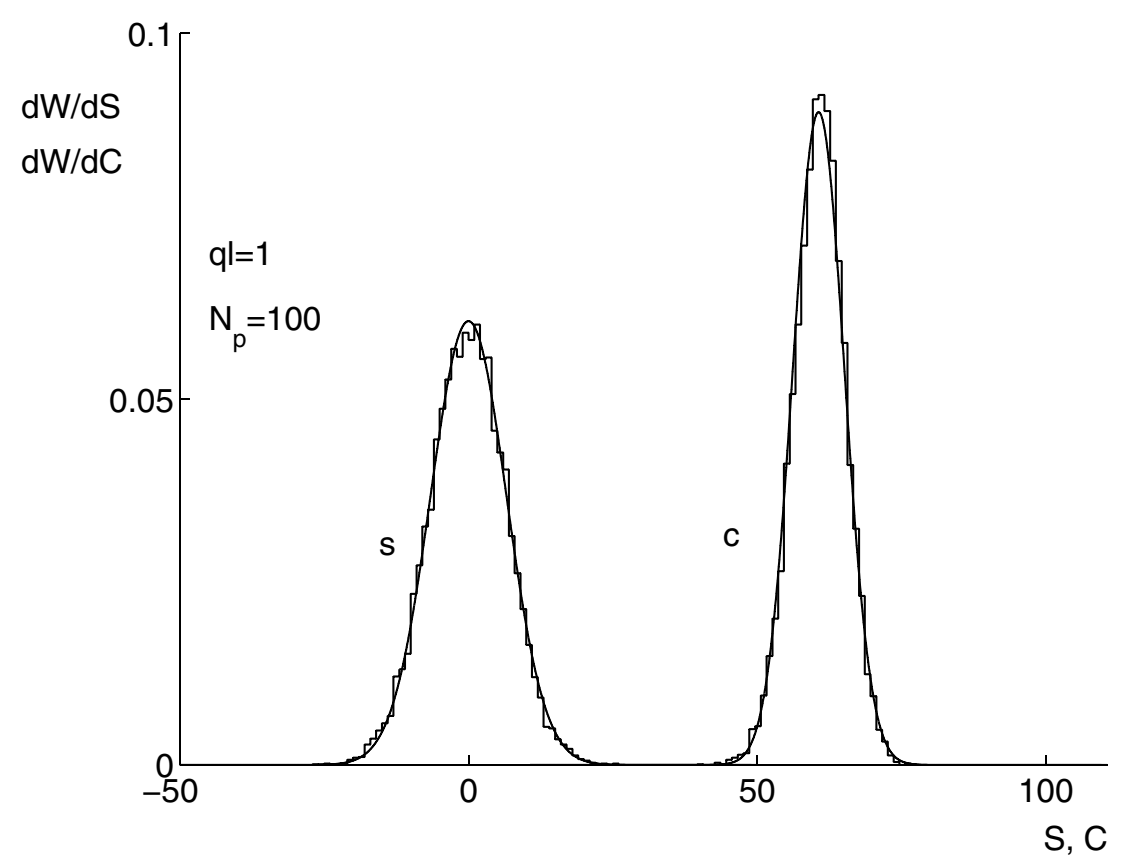

FIG. 4. A comparison of the analytical and numerical calculations for distributions (91) and (92). The curves $s$ and $c$ are given for distributions $d W / d S$ (91) and $d W / d C$ (92), respectively, at $q l=1, N_{p}=10^{2}$. The histograms represent results of numerical calculations for distribution of $S$ and $C$ defined in (89) for $10^{4}$ various sets of random numbers $\left\{z_{a} / l\right\}$.

$$
\begin{aligned}
\bar{c} & =\overline{\cos (q z)}=\int_{-\infty}^{+\infty} w(z) \cos (q z) d z=e^{-(q l)^{2} / 2} \\
\bar{s} & =\overline{\sin (q z)}=0, \quad \bar{c}^{2}=\overline{\cos ^{2}(q z)}=\frac{1}{2}\left(1+e^{-2(q l)^{2}}\right) \\
\bar{s}^{2} & =\overline{\sin ^{2}(q z)}=\frac{1}{2}\left(1-e^{-2(q l)^{2}}\right)
\end{aligned}
$$

We had performed the numerical calculations using the generator of random numbers from MATLAB. These calculations confirm the above distributions (91). It can be seen from Figs. 4 and 5, where the results of numerical calculations of $C$ and $S$ at $q l=1, N_{p}=10^{2}$ and $q l=$ $10, N_{p}=10^{3}$ are presented for $10^{4}$ various sets of $\left\{z_{a}\right\}$.

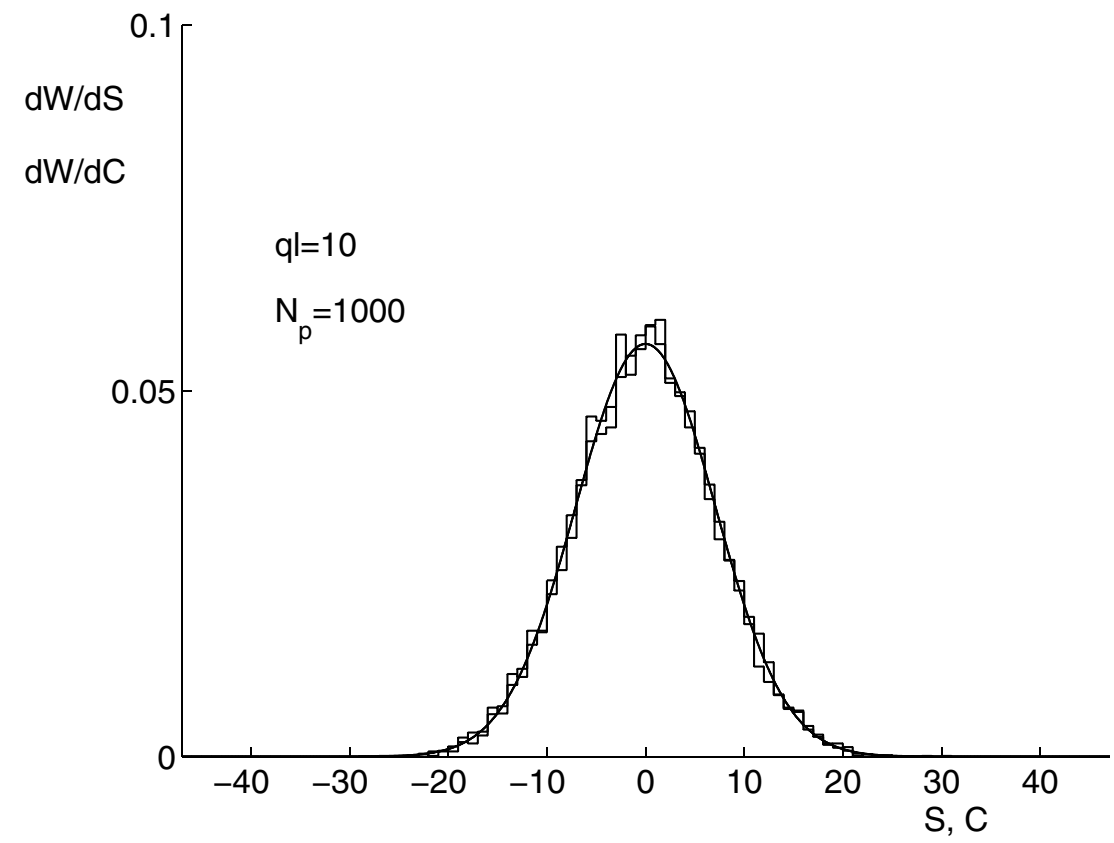

FIG. 5. The same as in Fig. 4, but for $q l=10, N_{p}=10^{3}$ (in this case the curves $s$ and $c$ practically coincide). 


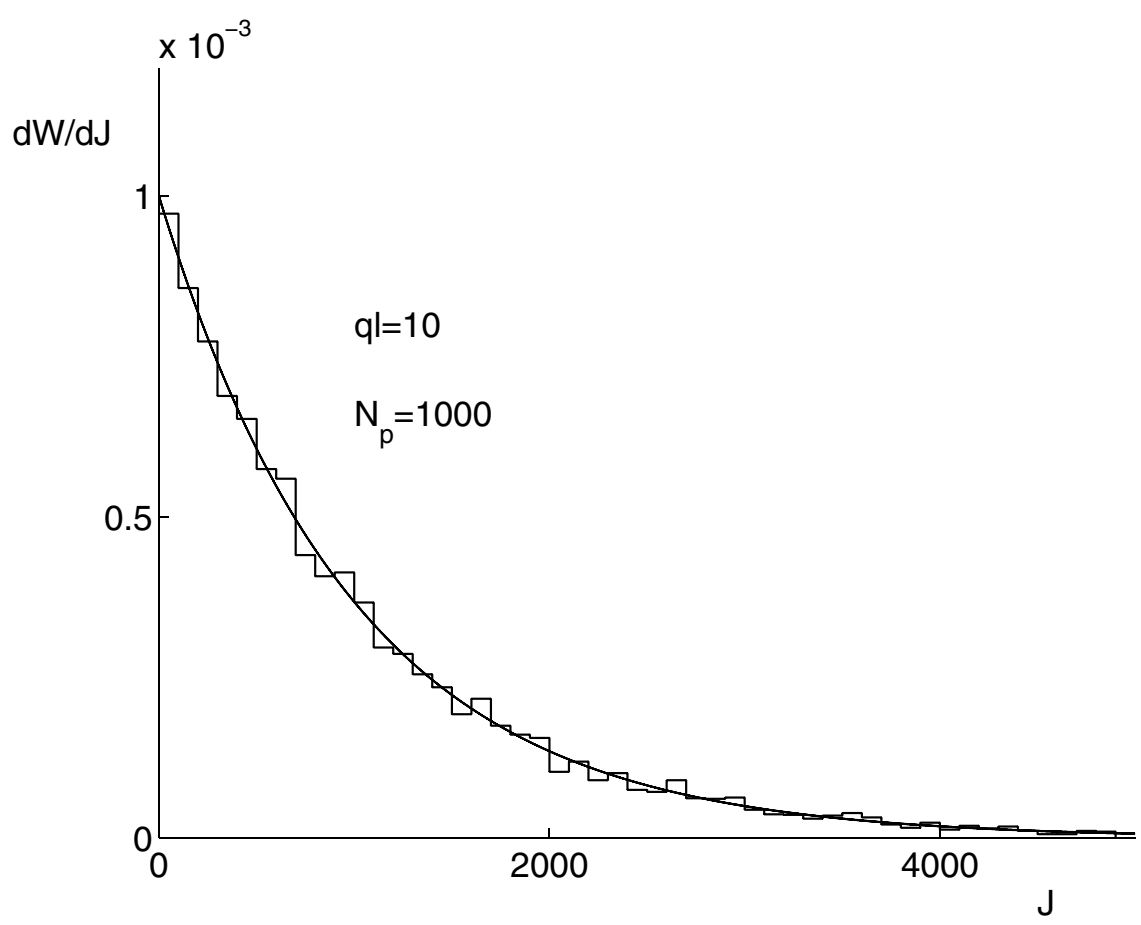

FIG. 6. A comparison of the analytical and numerical calculations for distributions (96). The curve is given for $d W / d J$ in accordance with Eq. (96) at $q l=10, N_{p}=10^{3}$. The histogram represents the result of numerical calculations for distribution of $J$ defined in (82) and (90) for $10^{4}$ various sets of random numbers $\left\{z_{a} / l\right\}$.

Since

$$
\begin{aligned}
& \langle C\rangle=N_{p} \bar{c}, \quad\left\langle\left(C-N_{p} \bar{c}\right)^{2}\right\rangle=N_{p}\left(\bar{c}^{2}-\bar{c}^{2}\right), \\
& \langle S\rangle=0, \quad\left\langle S^{2}\right\rangle=N_{p} \bar{s}^{2},
\end{aligned}
$$

we have

$$
\begin{aligned}
& \langle J\rangle=N_{p}+N_{p}\left(N_{p}-1\right) \bar{c}^{2}, \\
& \langle Z\rangle=N_{p}\left(N_{p}-1\right) \bar{c}^{2} \approx N_{p}^{2} \exp \left[-(\omega l / c)^{2}\right] .
\end{aligned}
$$

Moreover, taking into account that in the considered case $\left(N_{p} \bar{c}\right)^{2} \ll N_{p}$, we find that [in contrast to (84) and (85)]

$$
\langle J\rangle=N_{p}, \quad|\langle Z\rangle| \ll N_{p} .
$$

The distribution of the random quantity $J$ becomes very simple at $q l \gg 1$ :

$$
\frac{d W}{d J}=\frac{1}{N_{p}} e^{-J / N_{p}}
$$

The results of numerical calculations, presented in Fig. 6, confirm Eq. (96). It should be noted that distribution (96) is rather wide,

$$
\Delta J=\sqrt{\left\langle J^{2}\right\rangle-\langle J\rangle^{2}}=N_{p} ;
$$

therefore, the averaging over many various sets of $\left\{z_{a}\right\}$ is necessary to obtain the stable result for $\langle J\rangle$.

\section{SUMMARY AND DISCUSSION}

In the present paper we performed analysis of the coherent and incoherent contributions to bremsstrahlung in conditions (29) when the incoherent contribution dominates but large impact parameters give an essential contribution to the standard cross section. In this condition, the known correction (51) to the standard cross section is determined by the transverse distribution of particles in the beams.

We take into account the correlations of particles in the beam. The corresponding correction to the standard cross section is given by Eq. (52) and it is determined by correlations of particles in the transverse as well as in longitudinal coordinates.

We showed that replacing the sum $J(82)$ by the integral (83) in condition (29) is incorrect. As a consequence, the additional subtraction term, derived in Refs. [17,18], has to be omitted. On the other hand, such an additional subtraction should be taken into account for bremsstrahlung of ultrarelativistic electrons on oriented crystals. This conclusion is quite natural. A usual bunch at colliders can be considered as a gaseous media with a smooth particle distribution which has characteristic scales of the order of bunch sizes. In particular, the average particle density in such a bunch has the only scale in the longitudinal direction - the length of the bunch $l$. Therefore, the average field of the bunch has the spectral components in the region of frequencies $\omega=q_{z} c \sim c / l_{\text {coh }} \lesssim c / l$ and 
vanishes in the region of much higher frequencies considered here. On the contrary, in the crystal case there is another scale related to the size of the particle localization in the crystal structure.

Let us consider once again the experiments analyzed in Ref. [17].

The HERA experiment [12].-In this case $E_{e}=$ $27.5 \mathrm{GeV}$ and $l=8.5 \mathrm{~cm}$; therefore, the critical energy (45) is $E_{c}=27 \mathrm{keV}$. For the observed photon energies $E_{\gamma}=2-8 \mathrm{GeV}$, the parameter

$$
\frac{\omega l}{c} \sim \frac{E_{\gamma}}{E_{c}}>10^{4},
$$

and the coherent contribution is completely negligible. Therefore, the new correction to the previous results on the level of $10 \%$, obtained in [17], is, in fact, absent.

The VEPP-4 experiment [1].-In this case $E_{e}=$ $1.84 \mathrm{GeV}$ and $l=3 \mathrm{~cm}$; therefore, $E_{c}=0.34 \mathrm{keV}$. For the observed photon energies $E_{\gamma} \gtrsim 1 \mathrm{MeV}$, the parameter

$$
\frac{\omega l}{c} \sim \frac{E_{\gamma}}{E_{c}}>10^{3},
$$

and the coherent contribution is completely negligible.

Through the paper we consider the MD effect in bremsstrahlung. The MD effect for the $e^{+} e^{-}$pair production (for example, in the reaction $\gamma e \rightarrow e^{+} e^{-} e$ ) can be considered in the same manner-for details see Sec. 7.1 in Ref. [5].

\section{ACKNOWLEDGMENTS}

We are very grateful to A. Chernykh, V. Dmitriev, V. Fadin, I. Ginzburg, V. Katkov, I. Kolokolov, A. Milshtein, and N. Vinokurov for useful discussions. This work is supported in part by RFBR (Code No. 03-02-17734) and by the Fund of Russian Scientific Schools (Code No. 2339.2003.2)

\section{APPENDIX: AVERAGE PRODUCT OF PARTICLE DENSITIES IN THE BEAM}

In the classical limit (24) the average product of particle densities in the proton beam is given by the double sum over protons in the beam

$$
S\left(\mathbf{r}, \mathbf{r}^{\prime}\right)=\sum_{a, b=1}^{N_{p}}\left\langle\delta\left(\mathbf{r}-\mathbf{r}_{a}\right) \delta\left(\mathbf{r}^{\prime}-\mathbf{r}_{b}\right)\right\rangle .
$$

We split this expression into the sum with $a=b$ and the sum with $a \neq b$ :

$$
\begin{gathered}
S\left(\mathbf{r}, \mathbf{r}^{\prime}\right)=S_{1}+S_{2}, \quad S_{1}=\delta\left(\mathbf{r}-\mathbf{r}^{\prime}\right) \sum_{a}\left\langle\delta\left(\mathbf{r}-\mathbf{r}_{a}\right)\right\rangle, \\
S_{2}=\sum_{a \neq b}\left\langle\delta\left(\mathbf{r}-\mathbf{r}_{a}\right) \delta\left(\mathbf{r}^{\prime}-\mathbf{r}_{b}\right)\right\rangle .
\end{gathered}
$$

To perform the averaging, we introduce the average pro- ton distribution function

$$
f_{p}(\mathbf{r})=n_{p}(\mathbf{r}) / N_{p}
$$

with the normalization

$$
\int f_{p}(\mathbf{r}) d^{3} r=1
$$

It gives

$$
\left\langle\delta\left(\mathbf{r}-\mathbf{r}_{a}\right)\right\rangle=\int \delta\left(\mathbf{r}-\mathbf{r}_{a}\right) f_{p}\left(\mathbf{r}_{a}\right) d^{3} r_{a}=f_{p}(\mathbf{r}) .
$$

Note that quantity $\left\langle\delta\left(\mathbf{r}-\mathbf{r}_{a}\right)\right\rangle$ does not depend on index $a$ and, therefore,

$$
S_{1}=\delta\left(\mathbf{r}-\mathbf{r}^{\prime}\right) N_{p} f_{p}(\mathbf{r})=\delta\left(\mathbf{r}-\mathbf{r}^{\prime}\right) n_{p}(\mathbf{r}) .
$$

If we can neglect correlations between the particle coordinates, then the average product $\left\langle\delta\left(\mathbf{r}-\mathbf{r}_{a}\right) \delta\left(\mathbf{r}^{\prime}-\right.\right.$ $\left.\left.\mathbf{r}_{b}\right)\right\rangle$ for $a \neq b$ can be presented as the product of two averaged factors:

$$
\begin{aligned}
& \left\langle\delta\left(\mathbf{r}-\mathbf{r}_{a}\right) \delta\left(\mathbf{r}^{\prime}-\mathbf{r}_{b}\right)\right\rangle=\left\langle\delta\left(\mathbf{r}-\mathbf{r}_{a}\right)\right\rangle\left\langle\delta\left(\mathbf{r}^{\prime}-\mathbf{r}_{b}\right)\right\rangle \\
& \quad \text { for } a \neq b .
\end{aligned}
$$

As a consequence,

$$
\begin{aligned}
S_{2} & =\sum_{a \neq b}\left\langle\delta\left(\mathbf{r}-\mathbf{r}_{a}\right)\right\rangle\left\langle\delta\left(\mathbf{r}^{\prime}-\mathbf{r}_{b}\right)\right\rangle \\
& =N_{p}\left(N_{p}-1\right) f_{p}(\mathbf{r}) f_{p}\left(\mathbf{r}^{\prime}\right) .
\end{aligned}
$$

If we do not neglect the correlations between the particle positions, we should introduce the correlation function $C\left(\mathbf{r}, \mathbf{r}^{\prime}\right)$ as follows:

$$
S_{2}=\sum_{a \neq b}\left\langle\delta\left(\mathbf{r}-\mathbf{r}_{a}\right)\right\rangle\left\langle\delta\left(\mathbf{r}^{\prime}-\mathbf{r}_{b}\right)\right\rangle+C\left(\mathbf{r}, \mathbf{r}^{\prime}\right) .
$$

In that case we obtain instead of (A8) the expression

$$
S_{2}=N_{p}\left(N_{p}-1\right) f_{p}(\mathbf{r}) f_{p}\left(\mathbf{r}^{\prime}\right)+C\left(\mathbf{r}, \mathbf{r}^{\prime}\right)
$$

As a result,

$$
\begin{aligned}
S\left(\mathbf{r}, \mathbf{r}^{\prime}\right)= & N_{p}\left(N_{p}-1\right) f_{p}(\mathbf{r}) f_{p}\left(\mathbf{r}^{\prime}\right)+\delta\left(\mathbf{r}-\mathbf{r}^{\prime}\right) n_{p}(\mathbf{r}) \\
& +C\left(\mathbf{r}, \mathbf{r}^{\prime}\right) .
\end{aligned}
$$

Since in the right-hand side of this equation the first and second items usually do not compensate each other, we can use the approximation

$$
N_{p}\left(N_{p}-1\right) \approx N_{p}^{2}
$$

and, therefore,

$$
S\left(\mathbf{r}, \mathbf{r}^{\prime}\right)=n_{p}(\mathbf{r}) n_{p}\left(\mathbf{r}^{\prime}\right)+\delta\left(\mathbf{r}-\mathbf{r}^{\prime}\right) n_{p}(\mathbf{r})+C\left(\mathbf{r}, \mathbf{r}^{\prime}\right) .
$$

After that, the beam structure factor (27) is expressed as 


$$
\begin{aligned}
S\left(\mathbf{q}, \mathbf{q}^{\prime}\right) & =\sum_{a, b}\left\langle e^{-i\left(\mathbf{q r}_{a}-\mathbf{q}^{\prime} \mathbf{r}_{b}\right)}\right\rangle \\
& =F_{p}(\mathbf{q}) F_{p}^{*}\left(\mathbf{q}^{\prime}\right)+F_{p}\left(\mathbf{q}_{\perp}-\mathbf{q}_{\perp}^{\prime}\right)+C\left(\mathbf{q}, \mathbf{q}^{\prime}\right),
\end{aligned}
$$

where we take into account that $\mathbf{q}-\mathbf{q}^{\prime}=\mathbf{q}_{\perp}-\mathbf{q}_{\perp}^{\prime}$.

Let us now consider the distribution of the protons over longitudinal coordinates, described by exact $n(z)$ and average $n_{p}(z)$ densities defined in (60)-(62). In this case we can repeat the above derivation of Eq. (A13) for the longitudinal distribution with the result

$$
\left\langle n(z) n\left(z^{\prime}\right)\right\rangle=n_{p}(z) n_{p}\left(z^{\prime}\right)+\delta\left(z-z^{\prime}\right) n_{p}(z)+C\left(z, z^{\prime}\right),
$$

where $C\left(z, z^{\prime}\right)$ is the longitudinal correlation function. After that, the longitudinal beam structure factor is expressed as

$$
\int\left\langle n(z) n\left(z^{\prime}\right)\right\rangle e^{-i \omega\left(z-z^{\prime}\right) / c} d z d z^{\prime}=\left|F_{p}(\omega / c)\right|^{2}+C_{\omega}+N_{p},
$$

where the longitudinal form factor $F_{p}\left(q_{z}\right)$ is defined in (62) and

$$
C_{\omega}=\int C\left(z, z^{\prime}\right) e^{-i \omega\left(z-z^{\prime}\right) / c} d z d z^{\prime}
$$

If we introduce functions

$$
Z=\sum_{a \neq b} e^{-i \omega\left(z_{a}-z_{b}\right) / c}, \quad J=\sum_{a, b} e^{-i \omega\left(z_{a}-z_{b}\right) / c}=Z+N_{p}
$$

we can rewrite Eq. (A16) in the form

$$
\langle J\rangle=\langle Z\rangle+N_{p}=\left|F_{p}(\omega / c)\right|^{2}+C_{\omega}+N_{p} .
$$

[1] A. E. Blinov et al., Phys. Lett. 113B, 423 (1982).

[2] Yu. A. Tikhonov, Candidate thesis, INP Novosibirsk, 1982.

[3] V. N. Baier, V. M. Katkov, and V. M. Strakhovenko, Sov. Yad. Fiz. 36, 163 (1982).
[4] A. I. Burov and Ya. S. Derbenyev, INP Report No. INP 82-07, 1982.

[5] G. L. Kotkin, V. G. Serbo, and A. Schiller, Int. J. Mod. Phys. A 7, 4707 (1992).

[6] A. E. Blinov et al., Nucl. Instrum. Methods Phys. Res., Sect. A 273, 31 (1988).

[7] C. Bini et al., Nucl. Instrum. Methods Phys. Res., Sect. A 349, 27 (1994).

[8] G. L. Kotkin, S. I. Polityko, and V. G. Serbo, Sov. Yad. Fiz. 42, 692 (1985).

[9] G. L. Kotkin, S. I. Polityko, and V. G. Serbo, Sov. Yad. Fiz. 42, 925 (1985).

[10] G. L. Kotkin, S. I. Polityko, A. Schiller, and V. G. Serbo, Z. Phys. C 39, 61 (1988).

[11] G. L. Kotkin, E. A. Kuraev, A. Schiller, and V. G. Serbo, Phys. Lett. B 221, 96 (1989).

[12] K. Piotrzkowski, Z. Phys. C 67, 577 (1995).

[13] K. Melnikov and V. G. Serbo, Phys. Rev. Lett. 76, 3263 (1996); K. Melnikov, G. L. Kotkin, and V. G. Serbo, Phys. Rev. D 54, 3289 (1996).

[14] Y. Funakoshi, in Proceedings of the International Workshop on B-Factories: Accelerators and Experiments, 1993 (KEK, Tsukuba, 1993), p. 66.

[15] H. Burkhardt, in Proceedings of the 3rd Workshop on LEP Performance, Chamonix, 1993, edited by J. Poole (CERN, Geneva, 1993), p. 117; in Proceedings of the 7th Advanced Beam Dynamics Workshop, Dubna, 1995, edited by P. Beloshitsky and E. Perelstein (JINR, Dubna, 1996), p. 22; H. Burkhardt and R. Kleiss, in Proceedings of the 4th European Particle Accelerator Conference EPAC94. London, 1994, edited by V. Suller and Ch. Petit-Jean-Genaz (World Scientific, Singapore, 1994), Vol. 2, pp. 1353-1355.

[16] G. L. Kotkin and V. G. Serbo, Nucl. Instrum. Methods Phys. Res., Sect. A 517, 28 (2004); hep-ph/0212285.

[17] V. N. Baier and V. M. Katkov, Phys. Rev. D 66, 053009 (2002).

[18] V. N. Baier and V. M. Katkov, hep-ph/0305304.

[19] G. L. Kotkin and V. G. Serbo, hep-ph/0212102.

[20] V. B. Berestetskii, E. M. Lifshitz, and L. P. Pitaevskii, Quantum Electrodynamics (Pergamon, New York, 1994), 2nd English ed.

[21] I. F. Ginzburg, G. L. Kotkin, S. I. Polityko, and V. G. Serbo, Sov. Yad. Fiz. 55, 3310 (1992); 553324 (1992).

[22] R. Engel, A. Schiller, and V. G. Serbo, Z. Phys. C 71, 651 (1996). 